\title{
BEM/FEM FORMULATIONS FOR THE ANALYSIS OF PILES SUBMITTED TO HORIZONTAL LOADS
}

\author{
ENDI SAMBA LUAMBA \& JOÃO BATISTA DE PAIVA \\ Structures Department, São Carlos Engineering School, University of São Paulo, Brazil
}

\begin{abstract}
In this work, a comparative study of numerical formulations for the analysis of soil-pile interaction is presented. These formulations consist of the coupling of the Boundary Element Method (BEM) with the Finite Element Method (FEM). The pile may be subjected to horizontal or moment loading applied to its top. The soil is supposed to be a semi-infinite, elastic, isotropic continuum and is modeled by BEM. The pile is either represented by a single finite element or as a number of usual beam finite elements and the pile-soil interface tractions are represented by constant, linear, quartic and high-degree polynomial functions. Examples of individual piles subjected to horizontal loading are analyzed with the formulations presented, and the displacements and the diagram of the bending moments along the pile are compared.
\end{abstract}

Keywords: boundary element method, finite element method, finite difference method, pile-soil interaction.

\section{INTRODUCTION}

Soil-structure interaction is currently one of the problems that has received special attention from researchers in the most diverse research centers, mainly for its practical applications.

The piles of a foundation system beyond vertical loads are often subjected to high horizontal forces that can be caused by wind, sea waves, earth movement, etc.

Due to its complexity only with numerical methods the study of pile-soil interaction is possible. The numerical methods most used in this study are finite difference method, finite elements and boundary element method. The pile is usually modeled by Finite Difference Method (FDM) or Finite Element Method (FEM). Although the soil can also be modeled by the finite element method, the large number of elements required to properly represent the soil makes such analysis impracticable. Thus the soil is generally represented by the fundamental solutions of Mindlin [1] or Kelvin [2] or by others obtained analytically considering the soil formed by layers of different physical properties [3].

In relation to the study of soil-structure interaction in which the soil mass is represented by a continuous three-dimensional medium, several authors presented works such as Cheung and Nag [4], Poulos [5], Fatemi-Ardakani [6], Hemsley [7], Messafer and Coates [8], Matos Filho et al. [9] and many others.

With respect to the piles in Poulos [5] a formulation is presented for this study where the piles are analyzed using the beam theory and its differential equation is represented by finite differences. Another alternative is to analyze the pile using the finite element method. In this case the pile can be modeled as a single or several finite elements. The results for each pile representation vary from one to another. This work presents a comparative study of formulations for the analysis of the pile-soil interaction via BEM/ FEM coupling. The pile is subject to horizontal loading and bending moment and is modeled by the finite element method initially as a single element and the pile-soil interaction tractions are approximated by a cubic, quartic and high-degree polynomial functions. In sequence the pile is modelled by several usual beam finite elements and the interaction tractions adopted for each pile element are cubic, linear and concentrated forces at the nodes of the finite elements. The soil is modelled by the boundary element method and is assumed to be a 
semi-infinite continuum. Numerical results obtained with the various formulations are presented together.

\section{SOIL EQUATIONS}

The integral equation which relates the displacement $\mathrm{u}_{\mathrm{i}}$ at a generic point $s$ in the soil with the pile-soil interface tractions $p_{j}(s)$ is given by

$$
u_{i}=\int_{\Gamma} u_{i j}^{*}(p, s) p_{j}(s) d \Gamma(s), \quad(i, j=1,2,3),
$$

where $\Gamma$ is the pile-soil interface area.

The hyper singular boundary integral equation, when used, is given by:

$$
\mathrm{u}_{\mathrm{i}, \mathrm{x}_{3}}=\int_{\Gamma} \mathrm{u}_{\mathrm{ij}, \mathrm{x}_{3}}^{*}(\mathrm{p}, \mathrm{s}) \mathrm{p}_{\mathrm{j}}(\mathrm{s}) \mathrm{d} \Gamma(\mathrm{s}) \quad(\mathrm{i}, \mathrm{j}=1,2,3),
$$

where $\mathrm{u}^{*}{ }_{\mathrm{ij}}(\mathrm{p}, \mathrm{s})$ is Mindlin's [1] fundamental solution for the displacement at point $s$ in direction $j$ due to a unit load acting at point $p$ in direction $i$; $\mathrm{p}_{\mathrm{j}}$ is the pile traction in direction $j$ assumed to be a line-load into soil domain. In Appendix A the fundamental solution for horizontal displacements is presented.

Eqns (1) and (2) can be written as:

$$
\mathrm{u}_{\mathrm{i}}=\sum_{1}^{\mathrm{Ne}} \int_{\Gamma} \mathrm{u}_{\mathrm{ij}}^{*}(\mathrm{p}, \mathrm{s}) \mathrm{p}_{\mathrm{j}}(\mathrm{s}) \mathrm{d} \Gamma(\mathrm{s}), \mathrm{u}_{\mathrm{i}, \mathrm{x}_{3}}=\sum_{1}^{\mathrm{Ne}} \int_{\Gamma} \mathrm{u}_{\mathrm{ij}, \mathrm{x}_{3}}^{*}(\mathrm{p}, \mathrm{s}) \mathrm{p}_{\mathrm{j}}(\mathrm{s}) \mathrm{d} \Gamma(\mathrm{s}), \quad(\mathrm{i}, \mathrm{j}=1,2,3),
$$

where $\mathrm{Ne}$ is the number of line load (piles) immersed in the continuous medium. The approximating domain functions for the pile-soil interface tractions in the coupling BEM/ FEM are considered in two different ways. For the first each pile is represented by a single finite element and in this case the domain functions for tractions at the interface pile-soil for direction $\mathrm{X}_{1}$ are given by polynomials of degrees $3,4,5$, and 9 . These polynomials are written to represent the variation of the tractions along the pile. For the degree 4 polynomial besides the traction nodal values, the traction derivative $\left(\partial \mathrm{p} / \partial \mathrm{x}_{3}\right)$ is also used as a nodal parameter at the top of the pile. For this polynomial the hyper-singular displacement equation $(\partial \mathrm{u} 1 / \partial \mathrm{x} 3)$ is also being used. These functions for degrees $3,\{\varphi\}$ and $4\{\bar{\varphi}\}$ are given by:

$$
\{\varphi\}=\left\{\begin{array}{l}
\varphi_{1} \\
\varphi_{2} \\
\varphi_{3} \\
\varphi_{4}
\end{array}\right\}=\left\{\begin{array}{c}
-4.5 \xi^{3}+9 \xi^{2}-5.5 \xi+1 \\
13.5 \xi^{3}-22.5 \xi^{2}+9 \xi \\
-13.5 \xi^{3}+18 \xi^{2}-4.5 \xi \\
4.5 \xi^{3}-4.5 \xi^{2}+\xi
\end{array}\right\}\{\bar{\varphi}\}=\left\{\begin{array}{l}
\varphi_{1} \\
\varphi_{2} \\
\varphi_{3} \\
\varphi_{4} \\
\varphi_{5}
\end{array}\right\}\left\{\begin{array}{c}
-24.75 \xi^{4}+45 \xi^{3}-21.25 \xi^{2}+1 \\
-4.5 \xi^{4} L+9 \xi^{3} L-5.5 \xi^{2} L+\xi L \\
40.5 \xi^{4}-67.5 \xi^{3}+27 \cdot \xi^{2} \\
-20.25 \xi^{4}+27 \xi^{3}-6.75 \xi^{2} \\
4.5 \xi^{4}-4.5 \xi^{3}+\xi^{2}
\end{array}\right\},
$$

(a) cubic functions

(b) fourth degree function

with $\xi=z / L$ where $\xi$ is the dimensionless depth and $L$ is the total depth of the pile. In the second formulation the pile is divided into several finite beam elements and three other functions for the tractions in the directions $X_{1}$ along each element are considered. The first is a linear function and the second is a cubic function, the same one used to represent the displacements of the usual beam finite element. It is also considered as a third formulation 
that the interactions pile-soil tractions are concentrated forces applied at the nodes of the elements. The linear and cubic function are given by

$$
\left\{p_{s}\right\}=\left\{\begin{array}{c}
1-\xi \\
\xi
\end{array}\right\}^{T}\left\{\begin{array}{c}
p_{s i} \\
p_{s j}
\end{array}\right\} \quad\left\{p_{s}\right\}=\left\{\begin{array}{c}
1-3 \xi^{2}+2 \xi^{3} \\
\left(\xi-2 \xi^{2}+\xi^{3}\right) L \\
3 \xi^{2}-2 \xi^{3} \\
\left(-\xi^{2}+\xi^{3}\right) L
\end{array}\right\}^{T}\left\{\begin{array}{c}
p_{s i} \\
\left(\frac{d p_{s}}{d x_{3}}\right)_{i} \\
p_{s j} \\
\left(\frac{d p_{s}}{d x_{3}}\right)_{j}
\end{array}\right\}
$$
(a) linear function
(b) cubic function

where $p_{i,}\left(\partial p_{s} / \partial x_{3}\right)_{i}, p_{j},\left(\partial p_{s} / \partial x_{3}\right)_{j}$ are the nodal values of the interaction tractions and its derivatives at the nodes of the finite elements.

After performing all integrations in eqns (1) and (2) the following system of equations is obtained

$$
\left\{\mathrm{u}_{\mathrm{s}}\right\}=[G]\left\{\mathrm{p}_{\mathrm{s}}\right\},
$$

where $\left\{u_{s}\right\}$ is the vector of nodal displacements and $\left\{p_{s}\right\}$ is the vector with the nodal values of the interface tractions.

\section{SYSTEM OF EQUATIONS FOR THE PILE}

The pile is modelled by the finite element method in two deferent ways. First the pile is considered a single finite element with lateral displacements in direction $\mathrm{X}_{1}$ as nodal values and one nodal value for rotation about $\mathrm{X}_{2}$, illustrated in Fig. 1 for a polynomial with degree 4. The second the pile is divided into several usual beam finite element with nodes at its ends and with 4 nodal parameters, the horizontal displacements and their derivatives.

The potential energy of the pile is given by [10]

$$
\Pi_{\mathrm{ap}}=\frac{\mathrm{E}_{\mathrm{p}} \mathrm{I}_{\mathrm{p}}}{2} \int_{0}^{\mathrm{L}} \mathrm{u}^{\prime 2}(\mathrm{z}) \mathrm{dz}-\mathrm{F}_{1} \overline{\mathrm{u}}_{1}-\mathrm{M}_{1} \frac{\overline{\partial u_{1}}}{\partial x_{31}}+\int_{0}^{\mathrm{L}} \mathrm{P}_{\mathrm{x} 1}(\mathrm{z}) \mathrm{u}(\mathrm{z}) \mathrm{dz},
$$

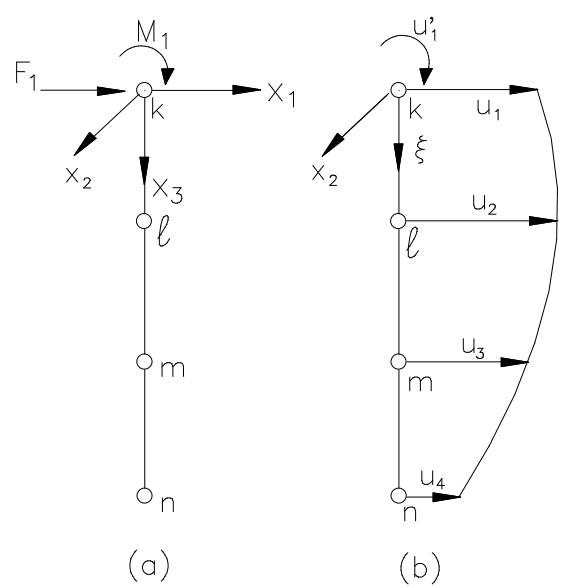

Figure 1: Model of the pile. (a) Forces at the top of the pile; (b) Nodal displacement parameters. 
where $E_{p}$ is the longitudinal modulus of elasticity of the pile; $I_{p}$ is the moment of inertia of the pile; $\mathrm{P}_{\mathrm{x} 1}$ is the horizontal traction along the pile, $\mathrm{F}_{1}$ and $\mathrm{M}_{1}$ are the horizontal load applied at the top of the pile, $\overline{\mathrm{u}_{1}}$ is the horizontal displacement at the top node of the pile and $\partial \overline{u_{1}} / \partial x_{3}$ is the derivative of the horizontal displacement at the top node of the pile.

Minimising the total potential energy the following system of equations can be obtained:

$$
\left[\mathrm{K}_{p}\right]\left\{\mathrm{u}_{\mathrm{p}}\right\}=\{\mathrm{F}\}+[\mathrm{Q}]\left\{\mathrm{P}_{\mathrm{p}}\right\}
$$

where $\left[K_{p}\right]$ is the stiffness matrix of the pile; $\left\{u_{p}\right\}$ is the vector of pile displacements, including rotations and lateral displacements; $\{F\}$ is the vector of equivalent nodal forces arising from external loads; $[Q]$ is the matrix that transforms element tractions to equivalent nodal forces; $\left\{P_{p}\right\}$ is the pile-soil interface traction vector.

\section{$4 \mathrm{BEM} / \mathrm{FEM}$ COUPLING}

Reordering the soil equations system (6) results in:

$$
\left\{\mathrm{p}_{\mathrm{s}}\right\}=[\mathrm{G}]^{-1}\left\{\mathrm{u}_{\mathrm{s}}\right\} \text {. }
$$

Taking account the force equilibrium that must hold along the interface between the pile and the continuous medium, viz.:

$$
\left\{\mathrm{p}_{\mathrm{s}}\right\}+\left\{\mathrm{p}_{\mathrm{p}}\right\}=0
$$

Eqns (15) and (16) can be combined to give:

$$
\left[\mathrm{K}_{\mathrm{c}}\right]\left\{\mathrm{u}_{\mathrm{p}}\right\}=\{\mathrm{F}\}-[\mathrm{M}]\left\{\mathrm{u}_{\mathrm{s}}\right\}
$$

where:

$$
[\mathrm{Q}][\mathrm{G}]^{-1}=[\mathrm{M}] \text {. }
$$

Now matrix $[\mathrm{M}]$ must be augmented to be of the same order as matrix $\left[\mathrm{K}_{\mathrm{c}}\right]$. Hence, if, for instance, the pile is modelled as a single element with cubic approximation for the tractions one columns of zeroes is added to [M], which stand for the neglected rotations about axes $\mathrm{X}_{2}$ in the soil. The enlargement of matrix [M] is denoted by the addition of a bar: $[\overline{\mathrm{M}}]$. As a consequence, the displacement vector in the soil equation must also be augmented:

$$
\left\{u_{s}\right\}^{T}=\left(u_{1} \frac{\partial u_{1}}{\partial x_{3}} u_{2} u_{3} u_{4}\right)
$$

If the traction's approximation is a quartic polynomial the hyper singular boundary integral equation of the displacement is used and so there is no need to add a column of zeroes

From the displacement compatibility, we have:

$$
\left\{\mathrm{u}_{\mathrm{s}}\right\}=\left\{\mathrm{u}_{\mathrm{p}}\right\}=\{\overline{\mathrm{U}}\},
$$

and so: 


$$
\left[\left[\mathrm{K}_{\mathrm{c}}\right]+[\overline{\mathrm{M}}]\right]\{\overline{\mathrm{U}}\}=\{\mathrm{F}\}
$$

Finally

$$
[\overline{\mathrm{K}}]\{\overline{\mathrm{U}}\}=\{\mathrm{F}\},
$$

in which $[\overline{\mathrm{K}}]$ is the final BEM/FEM coupling stiffness matrix; $\{\overline{\mathrm{U}}\}$ is the vector that encompasses all the nodal displacements of the system, including rotations; $\{F\}$ is the vector of external loads applied to the top of the pile (horizontal forces and bending moments).

\section{EXAMPLES}

In order to compare results obtained with various formulations, a reference formulation must be defined for this purpose. The formulation that provided the best results is that in which the pile is divided into several finite elements with linear approximation for the tractions at the interface with the soil. Results obtained with this formulation with the pile divided into 20 finite elements show excellent agreement with those presented by Poulos [5]. Initially, the results obtained with Poulos formulation and the BEM/FEM coupling for the pile shown in Fig. 2 are presented. The results presented are the displacements along the pile assuming it has a radius equal to $0.3048 \mathrm{~m}$ and its length is $6.096 \mathrm{~m}$. The inertia moment is $6.779 .10^{-3} \mathrm{~m}^{4}$, Epile $=21111000 \mathrm{kN} / \mathrm{m}^{2}$, Esoil $=211110 \mathrm{kN} / \mathrm{m}^{2}$ and the force applied at the top is $181.6 \mathrm{kN}$, the $\mathrm{X}_{1}$ direction. The Poisson ratio is 0.2 .

The results are presented with the following notations:

- BEM/FDM: Poulos Formulation.

- BEM/FEM 1EL: Formulation in which the pile is represented by a single finite element.

- BEM/FEM Linear: Formulation with the pile divided into finite elements and linear approximation for horizontal interface tractions.

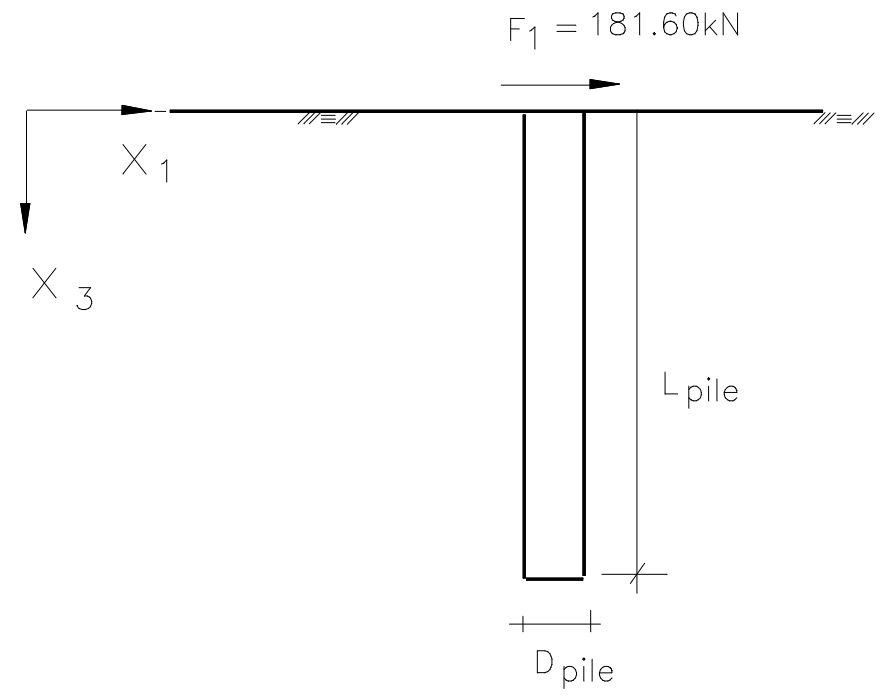

Figure 2: Pile data. 
- BEM/FEM Quartic: Formulation with the pile divided into finite elements and fourth degree polynomial approach for horizontal interface traction. In this formulation the hyper singular equation of the displacement is also used.

- BEM/FEM Hyper Singular: Formulation in which the pile is represented by a single finite element and fourth degree polynomial approach for horizontal interface traction. In this formulation the hyper singular equation of the displacement is also used.

In the Fig. 3 the results for pile of Fig. 2 are presented. The results obtained with BEM/FEM Linear are for a 9 finite element mesh. One can observe a good agreement between the results presented. However as the pile length increases, the BEM/FEM 1EL results are smaller than those obtained with BEM/FEM Linear as indicated in Fig. 4 for the same pile as the previous example now with $\mathrm{L}=12.184 \mathrm{~m}$. The results obtained with BEM/ FEM Linear with the pile divided into more than 18 finite elements are practically the same. These results show that the formulation BEM/FEM 1EL is more rigid than the BEM/ FEM Linear and that increasing the number of nodes along the length of the pile and using the formulation that considers the pile as a single finite element does not improve the finite element efficiency.

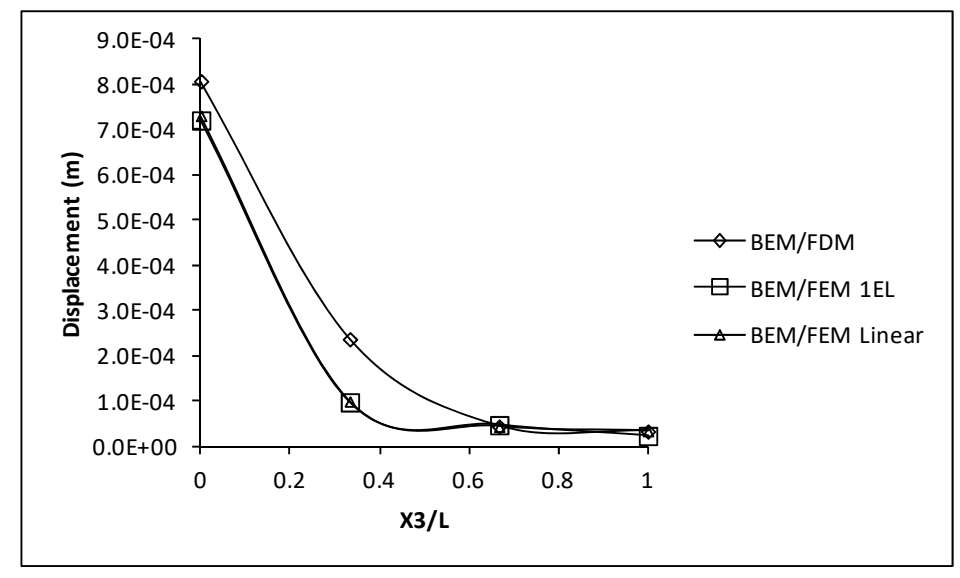

Figure 3: Displacement of the top of the pile.

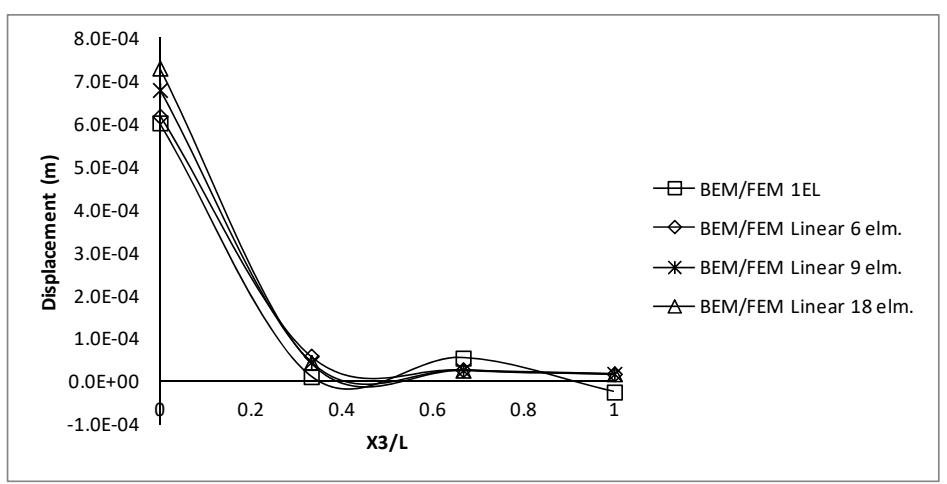

Figure 4: Displacement of the top of the pile obtained with BEM/FEM formulations. 
Next in Fig. 5 the results obtained for a pile of length $6.096 \mathrm{~m}$ are presented with the formulation that considers the pile a single element and with 4,5 and 9 nodes defined along its length. Also presented are the results obtained with the formulation in which the pile is divided into several finite elements, in this case considering a division of the pile into 20 elements of the same length.

It can be observed that increasing the degree of the polynomial leads to results that differ from those obtained with polynomial of lower degrees and with the results of the BEM/FEM.

Next Fig. 6 show the horizontal displacements of the pile of Fig. 2 now with a moment $\mathrm{M}=100 \mathrm{kNm}$ applied on its top.

The bending moment diagrams for the pile are shown in Fig. 7. It can be seen that increasing the number of nodes along the pile results in a worsening of the results.

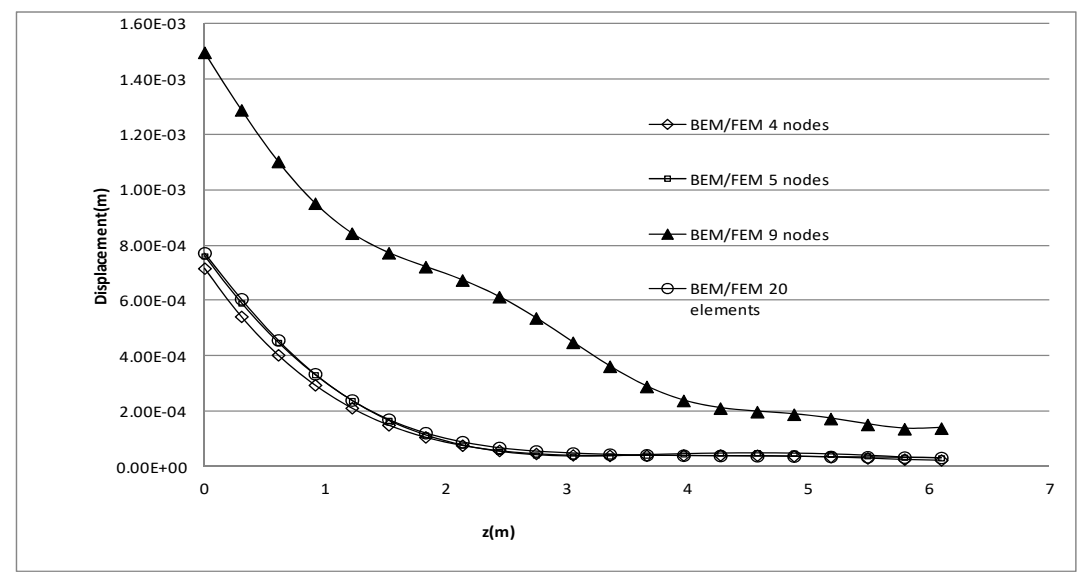

Figure 5: Displacement of the top of the pile obtained with BEM/FEM formulations.

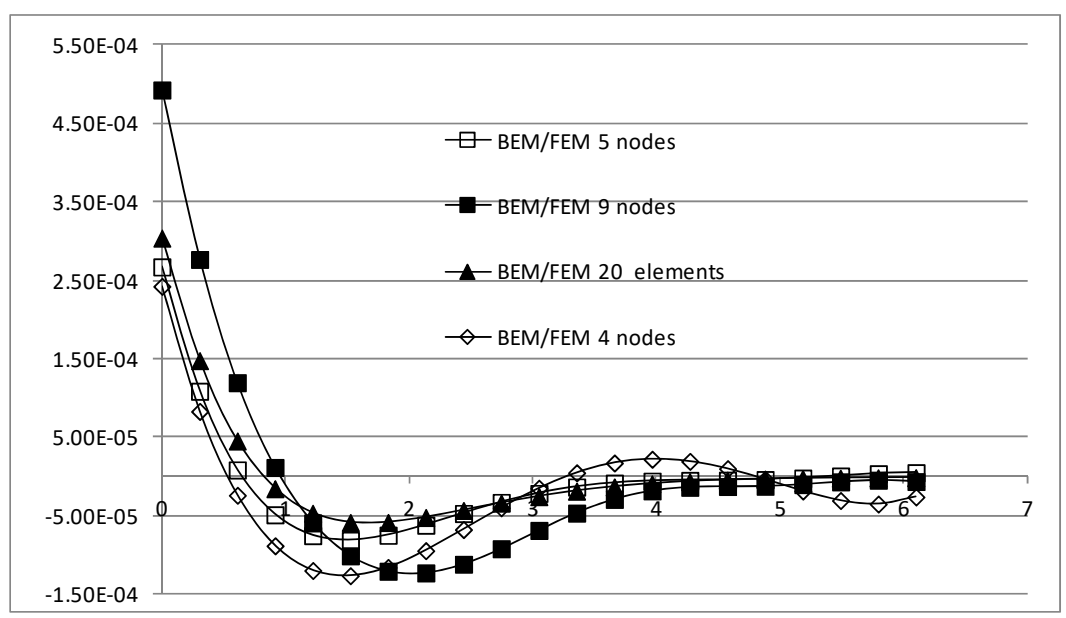

Figure 6: Horizontal displacement due to a concentrated bending moment applied on the top of the pile. 


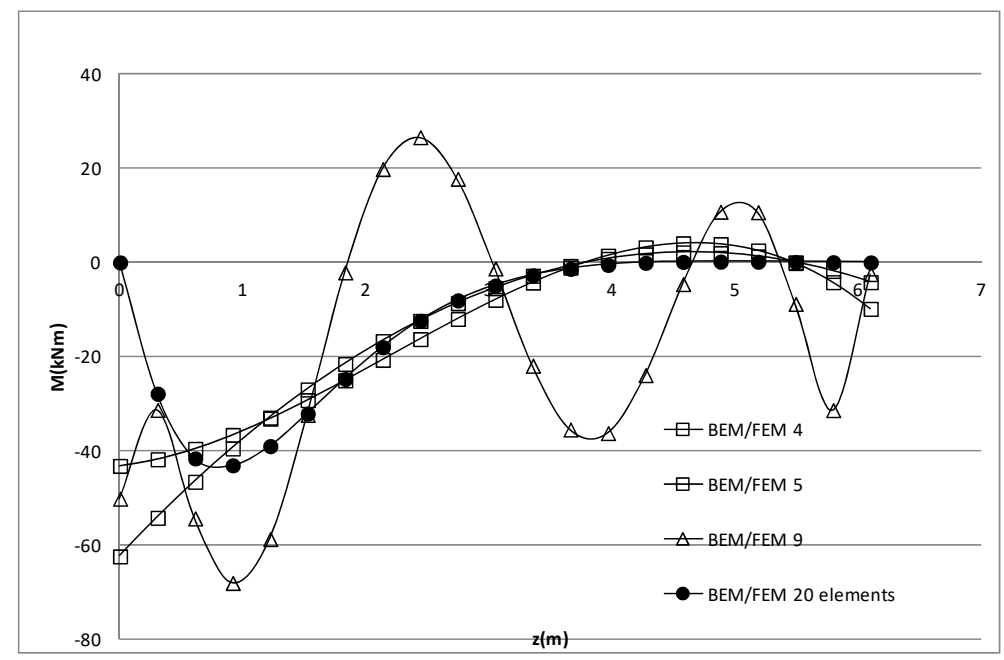

Figure 7: Bending moment diagram due to the horizontal force applied at the top of the pile.

Next example is same pile with $\mathrm{L}=6.098 \mathrm{~m}$ now considering the BEM/FEM Hyper Singular formulation in which the derivative of the displacement of the soil is also used compatibilizing rotations of the soil and the top of the pile and formulation BEM/FEM Quartic, which also uses the hyper singular equation for the soil compatibilizing pile and soil rotations at all finite elements nodes. The results of these analysis are shown in Fig. 8.

These results show that the use of the hyper singular equation makes the system more flexible and none of the presented results approaching those obtained with the other formulations indicated that the hyper singular equation is not suitable for analyzing this problem. The above results demonstrate that the modeling the pile with the usual finite beam element with linear approximation for the interaction tractions provide more consistent results.

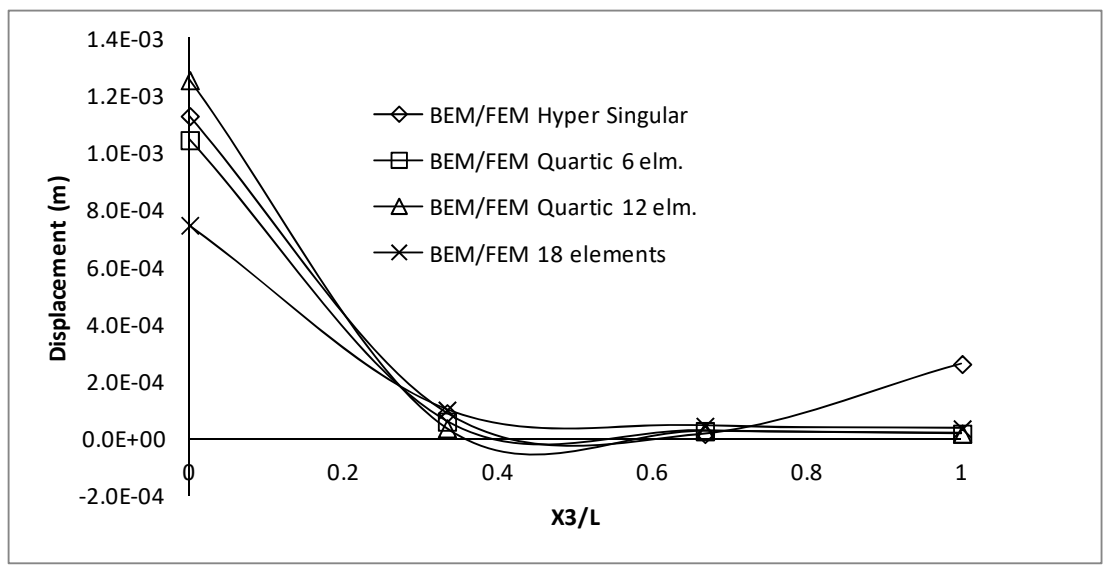

Figure 8: Displacement of the top of the pile obtained with BEM/FEM formulations. 
In the sequence are now compared the results obtained with two formulations in which the pile is divided into finite elements for usual beams and considering the interaction tractions linearly distributed or as concentrated forces at the nodes of the finite elements. Fig. 9 show the horizontal displacements along the pile shown in Fig. 2 now with $\mathrm{Lp}=20 \mathrm{~m}$. It can be seen that the two results are practically the same and that the punctual formulation is more flexible than the linear.

In Fig. 10 is presented the results obtained with the same formulations for the pile of Fig. 2 with length $\mathrm{L}=6.096 \mathrm{~m}$ varying the number of finite elements. The results show that both formulations give practically the same results.

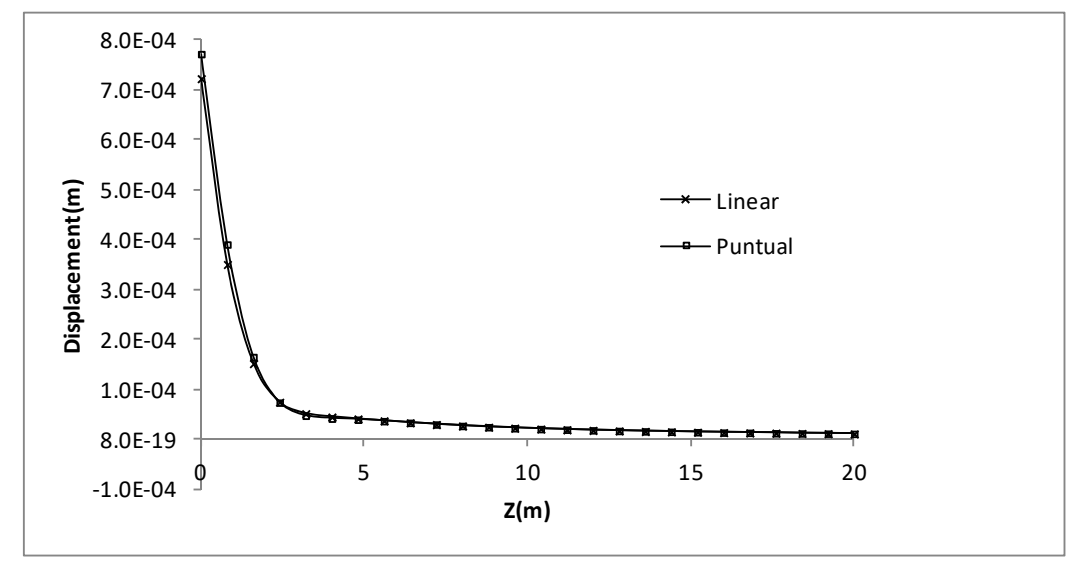

Figure 9: Displacements along the pile obtained with punctual and linear formulations.

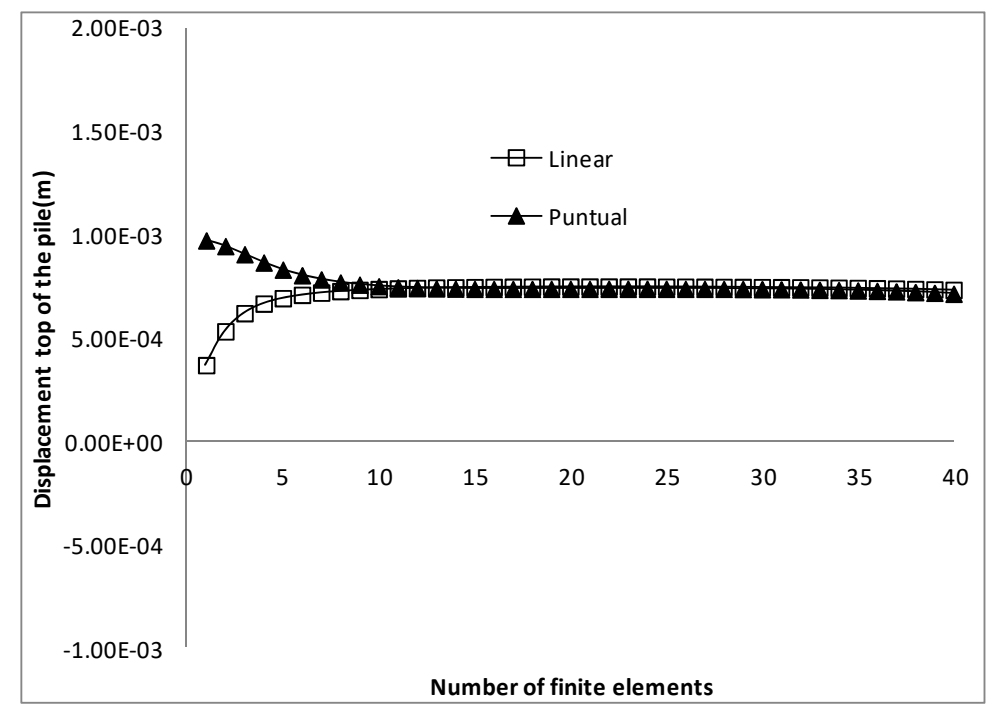

Figure 10: Displacements at the top of the pile with linear and punctual formulations as a function of the number of finite elements. 


\section{CONCLUSION}

In this paper a comparative study of numerical formulations for the analysis of pile-soil interaction with the pile subjected to horizontal loading is presented. The soil is represented by its integral equation and its respective derivative and the pile is modeled by finite difference method and the finite element method. The pile can be represented by a single finite element or several usual finite elements and the load on the interface is represented by linear or quartic functions and also by concentrated loads. Analyzing the results it can conclude the following.

The BEM/FEM coupling where the pile is represented by a single finite element the results for the short pile are in accordance with those obtained with other formulations however the model is more rigid than the others for long piles. When the pile is modeled by finite element with linear approximation for the traction in the pile-soil interface the results obtained show a convergence of results to a final value. When the interaction forces are approximated by concentrated forces the results are very close to those obtained with the linear approximation. However, the linear model becomes more suitable for analysis since this implies that the pile is continuously in contact with the soil while in the punctual approximation the pile is only in contact with the soil at the finite elements nodes.

The formulations that used the hyper singular displacement equation were more flexible than the others. From the presented studies it was concluded that the most suitable formulation for analyzing the interaction soil-pile where pile is subjected to horizontal loads is that the pile is divided into usual finite beam elements with linear approximation for the interface tractions.

\section{ACKNOWLEDGEMENT}

This work was carried out with $\mathrm{CNPq}$ support, the National Council for Scientific and Technological Development, Brazil.

\section{APPENDIX A: MINDLIN'S FUNDAMENTAL SOLUTION FOR HORIZONTAL DISPLACEMENTS}

Mindlin's fundamental solution for the horizontal displacements and its derivative at point $s$ due to a unit load acting at point $p$, Fig. 11 , is given by

$$
\begin{aligned}
& u_{11}^{*}=k d\left\{\frac{3-4 v}{r}+\frac{1}{R}+\frac{r_{1}^{2}}{r^{3}}+\frac{(3-4 v) r_{1}^{2}}{R^{3}}+\frac{2 C Z}{R^{3}}\left(1-\frac{3 r_{1}^{2}}{R^{2}}\right)+\frac{4(1-v)(1-2 v)}{R+R_{3}}\left[1-\frac{r_{1}^{2}}{R\left(R+R_{3}\right)}\right]\right\} \\
& \frac{\partial u_{11}^{*}(s, q)}{\partial x_{3}(s)}=k d\left\{\frac{(3-4 v) r_{3}}{r^{3}}-\frac{R_{3}}{R^{3}}+\frac{3 r_{1}^{2} r_{3}}{r^{5}}-\frac{3(3-4 v) r_{1}^{2} R_{3}}{R^{5}}+\frac{2 Z}{R^{5}}\left(r^{2}+Z^{2}-C Z-2 C^{2}\right)+\right. \\
& \left.-\frac{6 Z r_{1}^{2}}{R^{7}}\left(r^{2}+Z^{2}-3 Z C-4 Z^{2}\right)-\frac{4(1-v)(1-2 v)}{\left(R+R_{3}\right)^{2} R^{3}} r 1^{2}\left[R_{3}^{3}+R R_{3}^{2}-\left(2 R+R_{3}\right) r_{1}^{2}\right]\right\},
\end{aligned}
$$

where 


$$
\begin{aligned}
& k d=\frac{1+v}{8 \pi \cdot E(1-v)}, \quad r=\left(\begin{array}{ll}
r_{i} & r_{i}
\end{array}\right)^{1 / 2}, \quad R=\left(\begin{array}{ll}
R_{i} & R_{i}
\end{array}\right)^{1 / 2} \\
& r_{i}=x_{i}(q)-x_{i}(s), \quad R_{i}=x_{i}(q)-x_{i}\left(s^{\prime}\right), \quad C=x_{3}(s) \\
& Z=x_{3}(q) .
\end{aligned}
$$

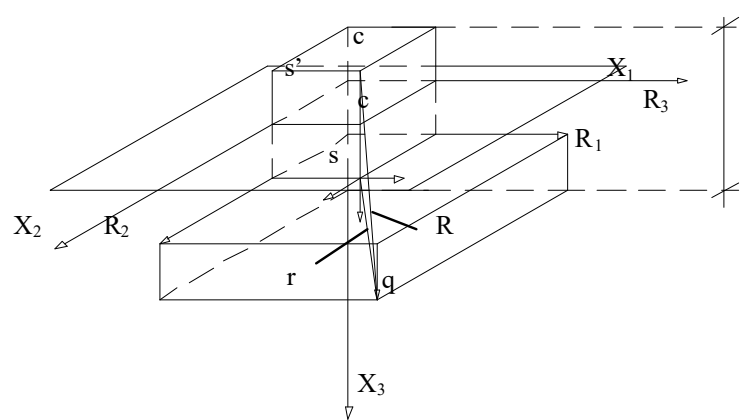

Figure 11: Mindlin problem.

\section{REFERENCES}

[1] Mindlin, R.D., Force at a point in the interior of a semi-infinite solid. Physics, 7, pp. 195-202, 1936.

[2] Kelvin, W.T., Note on the integrations of the equations of equilibrium of an elastic solid. Cambridge Dublin Math. J., 3, 1848.

[3] Ai, Z.H., Feng, D.L. \& Cheng, Y.C., BEM analysis of laterally loaded piles in multilayered transversely isotropic soils. Engineering Analysis with Boundary Elements, 37, pp. 1095-1106, 2013.

[4] Cheung, Y.K. \& Nag, D.K., Plates and beams on elastic foundations-linear and nonlinear behavior. Géotechnique, 18, pp. 250-260, 1968.

[5] Poulos, H.G., Behavior of laterally loaded piles I-Single piles. Journal of the Soil Mechanics and Foundations Division, ASCE, 97, SM5, pp. 711-731, 1971.

[6] Fatemi-Ardakani, B., A contribution to the analysis of pile-supported raft foundations. PhD thesis, Southampton University, Southampton, 1987.

[7] Hemsley, J.A., Elastic solutions for large matrix problems in foundation interaction analysis. Proc. Inst. Civ. Engrs., 89, pp. 471-494, 1990.

[8] Messafer, T. \& Coates, L.E., An application of FEM/BEM coupling to foundation analysis. Advances in Boundary Methods, 3, pp. 211-221, 1990.

[9] Matos Filho, R.F., Mendonça, A.V. \& Paiva, J.B., Static boundary element analysis of piles submitted to horizontal and vertical loads. Engineering Analysis with Boundary Elements, 29, pp. 195-203, 2005.

[10] Brebbia, C.A. \& Connor, J., Fundamentals of Finite Element Techniques, Butterworth \& Co Limited, 1973. 